\title{
Radiation-Induced Malignant Schwannoma (MPNST) of the Brachial Plexus
}

Keywords: MPNST (malignant peripheral nerve sheath tumour); Brachial plexus; Radiotherapy; Radionecrosis

\begin{abstract}
A MPNST tumour arising in the brachial plexus after an external radiation therapy is a rare complication and only a few cases are reported in the literature. We present a patient with a malignant neural sheath tumour of the brachial plexus and an intradural invasion along the foramen of C7. This complication arose 25 years after irradiation of a Hodgkin's lymphoma which had been definitely cured. The heralding symptoms anticipated of 5 months the spinal invasion and, due to the initial negativity of MRI, were misdiagnosed as a true postactinic palsy and initially treated with a pedicled fat wrapping of the upper plexus. After a two months-period of subjective improvement, a relentless severe progression of the palsy ensued, with excruciating pain and Horner syndrome. A PET-CT scan revealed an high glucose-consuming lesion and prompted to a new MRI, again negative for tumour around the plexus but showing an hyper intense mass at the level of the spinal cord. Similar experiences in the literature are briefly reviewed to identify the gold standard in the diagnosis and treatment of this unusual event.
\end{abstract}

\section{Introduction}

The occurrence of a malignancy after radiation therapy is a wellknown, although infrequent, long-term effect [1-5].

However, the Malignant Peripheral Nerve Sheath Tumours (MPNST) very uncommonly occurs as postirradiation neoplasms, the vast majority of them presenting in patients with neurofibromatosis type 1 . De novo appearance are highly unusual so as to localizations in the brachial plexus. A true post-irradiation MPNST of the brachial plexus has been reported in 9 cases, following radiotherapy for a breast cancer ( 5 cases) and Hodgkin lymphoma (4 cases). In 8 out of 9 cases it posed a direct unique challenge to life since the primitive disease had been by far definitely cured.

The time interval is very variable ( $2-41 \mathrm{yrs})$ and very long intervals are not unusual since the majority of the cases arose more than $20 \mathrm{yrs}$ after the irradiation. The time lag is probably unrelated to the quantity of the delivered irradiation, and has to do more with the capability of protection of the surrounding structures.

\section{This paper focuses on two main issues}

1) Raising awareness of this unusual complication in progressive brachial plexus palsies, especially those occurring a long time after irradiation and where one might easily consider the occurrence of radiation neuritis or a compressive neuropathy due to pectoralis minor muscle fibrosis.

At the very first stage of the disease, in fact, this kind of malignancy can be very difficult to differentiate and therefore a potentially fatal complication of external beam radiation might be missed.

2) Trying to identify, from the analysis of the literature, the gold

\section{Journal of}

Clinical \& Medical Case Reports

Ferraresi $\mathrm{S}^{1^{*}}$, Basso $\mathrm{E}^{1}$, Maistrello $\mathrm{L}^{1}$, Ballotta $\mathrm{MR}^{2}$ and Pavanato $\mathbf{G}^{3}$

${ }^{I}$ Department of Neurosurgery, Ospedale di Rovigo - Santa Maria della Misericordia, Italy

${ }^{2}$ Department of Pathology, Ospedale di Rovigo - Santa Maria della Misericordia, Italy

${ }^{3}$ Department of Radiotherapy, Ospedale di Rovigo - Santa Maria della Misericordia, Italy

\section{Address for Correspondence:}

Stefano Ferraresi, Department of Neurosurgery-Ospedale Santa Maria della Misericordia, Rovigo, Italy, Email: steveferraresi@gmail.com

Submission: 06 November, 2017

Accepted: 02 December, 2017

Published: 11 December, 2017

Copyright: (๑) 2017 Ferraresi S, et al. This is an open access article distributed under the Creative Commons Attribution License, which permits unrestricted use, distribution, and reproduction in any medium, provided the original work is properly cited.

standard for the treatment of such a lesion. Radical surgery and adjunctive radiation therapy are an option and, in affirmative case, to what extent and with which benefits?

\section{Case Report}

This 48-year old male presented with a slow occurring palsy of the left brachial plexus showing impairment of shoulder abduction and of biceps function, along with moderate pain in the arm and forearm. A MRI centered on the brachial plexus disclosed no abnormalities.

Due to an external radiation therapy (30 Gy in a 4 weeks time plus chemiotherapy) done 25 years before for an Hodgkin lymphoma and the negativity for tumour infiltration at MRI, a diagnosis of postactinic brachial plexus palsy was posed.

Surgery was undertaken: the upper plexus appeared abnormally pale but no infiltration was visible neither of the paraneurium nor of the epineurium. The tendon of the pectoralis minor was detached and a complete scalenectomy with decompression of the supraclavicular plexus and the subclavian artery was followed by wrapping of the upper trunk with a pedicled fat flap rotated from the pectoral region.

The patient did well, pain and the motor deficit seemed to have stabilized for the following three months. Later on, however, he experienced progression of the disease with near complete plexus palsy (some residual wrist and finger flexion), excruciating pain and Horner syndrome (Figures 1a and 1b).

A PET-CT scan disclosed a hypercapting area along the plexus extending to the cervical spinal area (Figures $2 a$ and $2 b$ ). An MRI was again negative for tumour in the plexus but a cervical intradural 1,5 $\mathrm{cm}$ diameter node was present (Figures $3 \mathrm{a}$ and $3 \mathrm{~b}$ ).

The patient was rapidly readdressed to surgery and we totally removed a well-defined mass easily separated from the spinal cord. The tumour was in continuity with the ventral and dorsal rootlet of C7. The root was removed and the dural sleeve of C7 sealed with two 
Citation: Ferraresi S, Basso E, Maistrello L, Ballotta MR, Pavanato G. Radiation-Induced Malignant Schwannoma (MPNST) of the Brachial Plexus. J Clin Med Case Reports. 2017;4(2): 3.

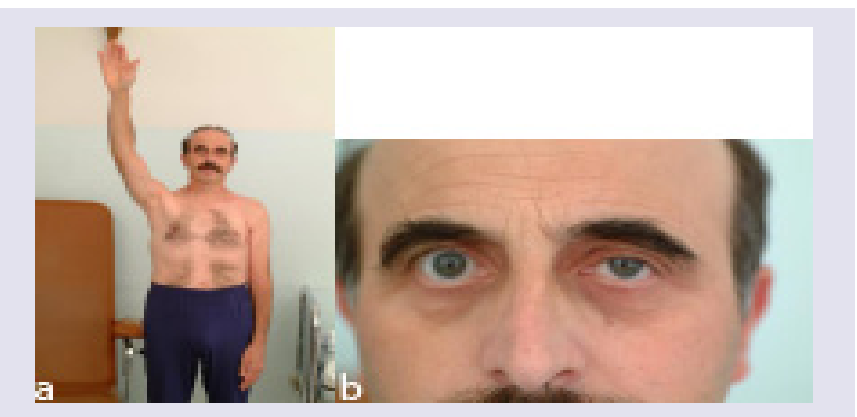

Figure 1a,b: Subtotal left brachial plexus palsy with left Horner syndrome.

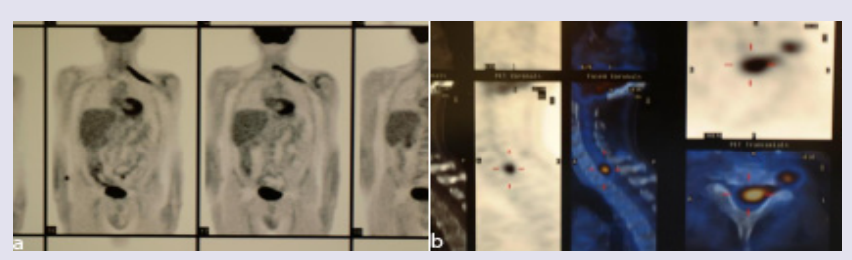

Figure 2: (a) PET scan demonstrating an involvement of the plexus and (b) their spinal counterpart.

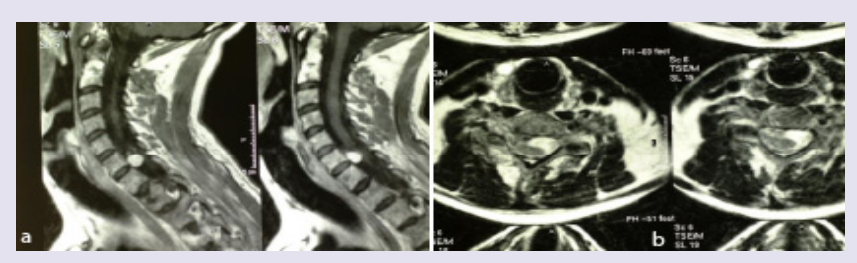

Figure 3: Contrast-enhanced MRI demonstrating a spinal extension of a tumour of C7 (a-sagittal; b- axial).

ligating titanium clips and excluded from the intradural space. The postoperative course was uneventful. A CT scan of the chest and of the abdomen disclosed no abnormalities. A highly invasive removal of the entire plexus was proposed and rejected by the patient. He died 13 months later with diffuse pulmonary disease. No adjuvant therapy was instituted.

\section{Histological findings}

The initial hypothesis of a recurrence of the Hodgkin disease is clearly excluded. Histologically the tumour is highly cellular and composed of spindle cells disposed in fascicles. The elongate tumor cells show cytologic atypia, hyperchromatic nuclei and moderate amounts of faintly eosinophilic cytoplasm (Figures 4a-4c).

A mitotic index of 2-3 per 10 high-power fields is seen. Tumor necrosis is absent. Immunohistochemical stains show positivity for S-100 and Vimentin and negativity for EMA. Expression of MIB1Ki67 is high (30\%) (Figures 5 and 6).

A diagnosis of primary malignant tumour of peripheral nerves (MPNST) is straightforward.

\section{Discussion}

RT-induced soft tissues sarcomas are rare, and only about $5 \%$ of these are MPNST [6]. Given the rarity of their occurrence, however, very little is known about these tumors.

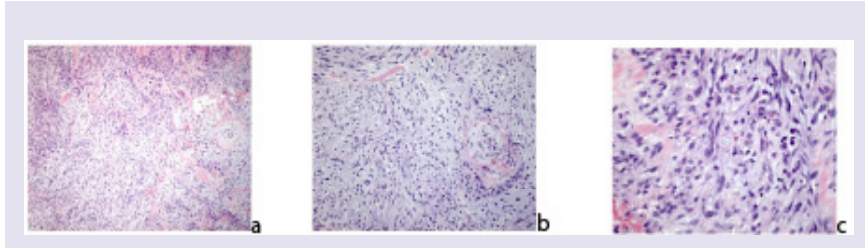

Figure 4 a-c: MPNST is composed of highly cellular, spindle cell proliferation. The cells are disposed in sweeping fascicles, the nuclei are hyperchromatic (EE x 100).

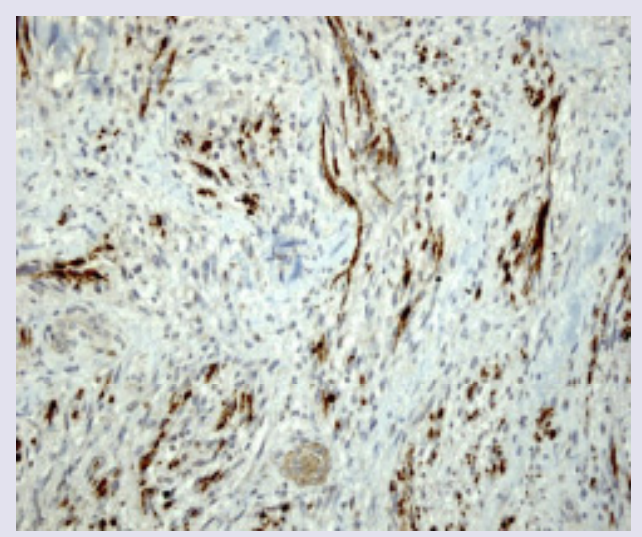

Figure 5: S-100 protein represents the most useful marker of nerve sheath differentiation (x 200).

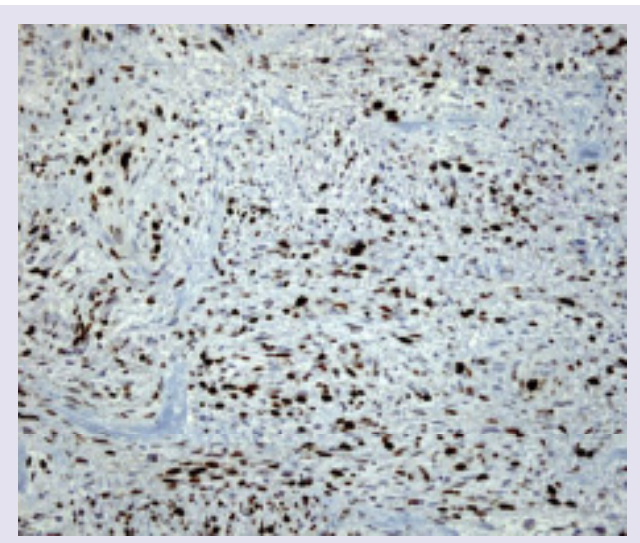

Figure 6: Ki-67-MIB1 immunoreactivity: high growth fraction (x 200).

MPNST is a very peculiar tumour, commonly included among sarcomas. However, a sarcoma is the malignant counterpart of a connective tissue tumour (bone, muscle, cartilage) which the nerve is not. The neural tumours are a derivation from the ectodermal crest, therefore Schwann cells are more akin to the melanocytes and so should be with regard to their malignant evolution.

Anyway, the risk of MPNST after RT is difficult to estimate, but has been reported as $0.06 \%$, most commonly following exposure to external beam radiation in the setting of breast cancer or lymphoma [7].

The retrospective analysis of the few reported cases of RTinduced tumours of the brachial plexus ( 1 case, 5 cases, 1 case, 2 cases, the one in the posterior triangle of the neck reported by Newbould is 
Citation: Ferraresi S, Basso E, Maistrello L, Ballotta MR, Pavanato G. Radiation-Induced Malignant Schwannoma (MPNST) of the Brachial Plexus. J Clin Med Case Reports. 2017;4(2): 3.

ISSN: $2332-4120$

uncertain) shows that breast tumour and lymphoma are the primitive diagnoses involved in the reported cases of radiation induced brachial plexus MPNST $[2,4,8-10]$. It also points to the fact that the time intervals between irradiation and onset of a MPNST, although very variable (2-41 yrs), tend to be rather long.

Unexpectedly long intervals are not unusual since more than half of the patients came to medical attention over $20 \mathrm{yrs}$ after the irradiation. The incidence of tumours is not dose-dependent with certainty, although, from the general experience, higher dosages are usually related to a higher rate of complications. As a matter of facts, nowadays, the patients show in overall a less and more fractionated dosage of radiation. This could turn into an even lower incidence of these cases in the future.

The peculiarity of this case is the misleading appearance of the plexus which looked absolutely not infiltrated nor enlarged, as it happens, on the contrary, in the vast majority of the malignant brachial plexus tumour cases.

Here, the pain and the progression of the plexus palsy, coupled with the surgical finding and the initially negative neuroimaging, led our senior surgeon (S.F.) to a diagnosis of true post-actinic plexitis. Differently from what is generally thought by the oncologists, this is an extremely rare occurrence if compared to the overwhelming majority of recurrences of malignant tumour cells infiltrating the neural sleeves, as occurs, for example, in late breast cancer plexopathies.

A more detailed neurophysiological diagnosis with repetitive nerve stimulation (a diagnostic aid for distinguishing cervical spondylotic amyotrophy from amyotrophic lateral sclerosis) was not undertaken $[11,12]$. Both diagnoses have been discarded because of the pain (absent in ALS) and the association of Horner syndrome, which in turn points to a compressive cause located outside the neuroforamen, when not in the lung apex.

The hypothesis of a malignancy is often overlooked because many years have elapsed from the time of irradiation and a recurrence of the primitive disease is excluded. Therefore we could readily state that a very long time interval (at the latest 41 -years) may eventually be a reason to discard a local recurrence of the primitive disease but not to dismiss the possibility of a neoplasm.

Concerning outcome, the most omnicomprehensive work has been done by LaFemina and coworkers [6]. They analyze the survival rates of RT-induced MPNSTs compared to NF1 associated and sporadic cases and conclude that the first carry the worst prognosis.

\section{Conclusion}

Even if the literature is quite poor of malignant tumours of the brachial plexus induced by a former radiation, some ideas are emerging from our experience and that of the literature.

Immediate recognition and treatment of radiation induced tumors is crucial because they tend to grow rapidly and have a low response to conventional radiation and chemotherapy.

However, it is not always easy to recognize them.

The clinical presentation may mimic a benign disease, which in turn may lead to a wrong diagnosis and an incomplete surgical approach aimed only at decompression of the neural structures.

Even if the radiological and pathological findings are negative or uncertain, in the presence of an history of previous radiotherapy, one must insist and get more and more findings at short and regular intervals.

This in spite of the general disagreement, when not open mistrust, which often accompanies this attitude, shown particularly by the radiological counterpart.

Nevertheless the common and rather rapid fatal outcome of these tumours should prompt a high diagnostic suspicion index, with the liberal use of repeated neuroimaging and, when the tumour is found, an aggressive therapeutic attitude. Surgery is the main tool in these cases especially because no clear survival benefit has been established after a new cycle of Radiotherapy or chemotherapy $[6,9,13,14]$.

\section{References}

1. Dawes B, Clark J, Byrne ST, Kalnins R, Gonzalvo A, et al. (2014) Radiation induced malignant peripheral nerve heath tumour of the second cervical nerve. J Clin Neurosci 21: 1990-1993.

2. Ducatman BS, Scheithauer BW, Piepgras DG, Reiman HM, Ilstrup DM (1986) Malignant peripheral nerve sheath tumors. A clinicopathologic study of 120 cases. Cancer 57: 2006-2021.

3. Falavigna A, da Silva PG, Teixeira W (2016) Radiotherapy-induced tumors of the spine, peripheral nerve, and spinal cord: case report and literature review. Surg Neurol Int 7 (Suppl 4): S108-S115.

4. Foley KM, Woodruff JM, Ellis, FT, Posner JB (1980) Radiation-induced malignant and atypical peripheral nerve sheath tumors. Ann Neurol 7: 311318.

5. Jaeckle KA (2010) Neurologic manifestations of neoplastic and radiationinduced plexopathies. Semin Neurol 30: 254-262.

6. LaFemina J, Qin LX, Moraco NH, Antonescu CR, Fields RC, et al. (2013) Oncologic outcomes of sporadic, neurofibromatosis-associated, and radiation-induced malignant peripheral nerve sheath tumors. Ann Surg Oncol 20: 66-72.

7. Mavrogenis AF, Pala E, Guerra G, Ruggieri P (2012) Post-radiation sarcomas Clinical outcome of 52 Patients. J Surg Oncol 105: 570-576.

8. Hussussian CJ, Mackinnon SE (1999) Postradiation neural sheath sarcoma of the brachial plexus: a case report. Ann Plast Surg 43: 313-317.

9. Newbould MJ, Wilkinson N, Mene A (1990) Post-radiation malignant peripheral nerve sheath tumour: a report of two cases. Histopathology 17 : 263-265.

10. Zadeh G, Buckle C, Shannon P, Massicotte EM, Wong S, et al. (2007) Radiation induced peripheral nerve tumors: case series and review of the literature. J Neurooncol 83: 205-212.

11. Zheng C, Jin X, Zhu Y, Lu F, Jiang J, et al. (2017) Repetitive nerve stimulation as a diagnostic aid for distinguishing cervical spondylotic amyotrophy from amyotrophic lateral sclerosis. Eur Spine J 26: 1929-1936.

12. Zheng C, Zhu Y, Lv F, Ma X, Xia X, et al. (2014) Abnormal flexor carpi radialis $\mathrm{H}$-reflex as a specific indicator of $\mathrm{C} 7$ as compared with $\mathrm{C} 6$ radiculopathy. $J$ Clin Neurophysiol 31: 529-534.

13. Sordillo PP, Helson L, Hajdu SI, Magill GB, Kosloff C, et al. (1981) Malignant schwannoma--clinical characteristics, survival and response to therapy. Cancer 47: 2503-2509

14. Wong WW, Hirose T, Scheithauer BW, Schild SE, Gunderson LL (1998) Malignant peripheral nerve sheath tumor: analysis of treatment outcome. Int J Radiat Oncol Biol Phys 42: 351-360. 